González-Fernández, D., Iturra Herrera, C. y Hernández González, O. (2022). Trabajo colaborativo entre maestros y logopedas: Una revisión acerca de las barreras y de su estructura subyacente. Revista de Investigación Educativa, 40(1), 165-182.

DOI: http://dx.doi.org/10.6018/rie.444821

\title{
Trabajo colaborativo entre maestros y logopedas: Una revisión acerca de las barreras y de su estructura subyacente
}

\section{Collaborative work between teachers and speech therapists: A review about the barriers and their underlying structure}

\author{
Daniela González-Fernández*1, Carolina Iturra Herrera** y Osvaldo Hernández González* \\ *Doctorado en Ciencias Humanas, Facultad de Psicología e Instituto de Estudios Humanísticos Juan Ignacio Molina, \\ Universidad de Talca, Talca, Chile \\ **Facultad de Psicología, Universidad de Talca, Talca. Chile
}

\begin{abstract}
Resumen
Introducción: La colaboración entre maestros y logopedas permite un abordaje inclusivo de las necesidades específicas de apoyo educativo nivel de aula. A pesar de la importancia del trabajo colaborativo entre ambos profesionales, existe evidencia de que diversas barreras limitan su desarrollo. El objetivo de esta revisión es identificar las barreras que han descrito maestros y logopedas para el desarrollo de trabajo colaborativo. Para posteriormente organizarlas siguiendo el modelo ecológico. Método: Se realizó una revisión sistemática siguiendo las directrices entregadas por PRISMA. Se buscaron artículos científicos indexados en bases de datos Web of Science, Proquest, Ebsco, Scopus. Se utilizaron palabras claves, seleccionando aquellas investigaciones que cumplieron con criterios de inclusión. Resultados: Los resultados muestran que existen barreras, distribuidas en tres sistemas interconectados, micro, meso y macrosistema. Discusión y Conclusiones: Se discuten posibles acciones que facilitarían el desarrollo del trabajo colaborativo. Formuladores de políticas educativas que apuntan a una educación más inclusiva y efectiva podrían considerar los hallazgos de este estudio, realizando intervenciones focalizadas en los colegios para favorecer la colaboración entre maestros y logopedas.

Palabras clave: interacción; logopeda; maestro; barreras
\end{abstract}

1 Correspondencia: Daniela González-Fernández, dangonzalez@utalca.cl, 3460000, becaria ANID N5031/2019. Este trabajo fue apoyado por el Programa de Investigación (PIA) en Ciencias Cognitivas, Centro de Investigación en Ciencias Cognitivas, Facultad de Psicología, Universidad de Talca. Talca. Chile. 


\begin{abstract}
Introduction: Collaboration between teachers and speech therapists enables an inclusive approach to special educational needs in the classroom. Despite the importance of collaborative work between both professionals, there is evidence that various barriers limit their development. The objective of this review is to identify the barriers that teachers and speech therapists have described for the development of collaborative work and to organize them following the ecological model. Method: A systematic review was carried out following the guidelines provided by PRISMA. We searched for scientific articles indexed in Web of Science, Proquest, Ebsco, Scopus. Keywords were used, selecting those studies that met the inclusion criteria. Results: The results show that there are barriers, distributed in three interconnected systems: micro, meso and macrosystem. Discussion and Conclusions: Possible actions that would facilitate the development of collaborative work are discussed. Those who are responsible for educational policy could consider the findings of this study, carrying out specific interventions in schools for educational inclusion and collaboration between teachers and speech therapists.

Keywords: interaction; speech and language therapist; teacher; barriers
\end{abstract}

\title{
Introducción
}

La educación inclusiva ha planteado que la diversidad del alumnado existente en las aulas debe ser tomada como una oportunidad que permita enriquecer el proceso de aprendizaje (United Nations Educational, Scientific and Cultural Organization [UNESCO], 2015; Ley orgánica para la mejora de la calidad educativa [LOMCE], 2013). Así, esta educación tiene como desafío dar respuestas a las distintas necesidades específicas de todos los alumnos (Ainscow, 2017; UNESCO, 2015; LOMCE, 2013). Para ello, lineamientos internacionales orientan y promueven que todas las personas se eduquen y participen plenamente de la sociedad (Unión Europea, 2017), sobre todo el alumnado que presenta necesidades específicas en las que la comunidad educativa debe asegurar el buen desarrollo del proceso de aprendizaje (Agencia Europea para el Desarrollo de la Educación con Necesidades Educativas Especiales [AEDNEE], 2011a). En dicho proceso, cobra relevancia la participación de distintos profesionales de la educación, que deben colaborar entre sí para que conjuntamente, puedan dar respuestas a las demandas cognitivas y emocionales de todos sus alumnos (American Speech-Language-Hearing Association [ASHA], 2016).

Uno de los profesionales que apoya al maestro en el proceso de aprendizaje es el logopeda, quien focaliza sus esfuerzos para trabajar con estudiantes que presentan dificultades en el desarrollo lenguaje, como por ejemplo, la conciencia fonológica (Acosta et al., 2010; Koutsoftas et al., 2009; Moreno et al., 2012) o el vocabulario (Axpe et al., 2017; Moreno et al., 2012; Murphy et al., 2016; Steele \& Mills, 2011; Wright et al., 2018). No obstante, para lograr desarrollar un trabajo conjunto en un contexto inclusivo, tanto el logopeda como el maestro deben asumir una posición de cooperación, lo que implica adoptar un rol distinto al tradicionalmente desempeñado bajo los enfoques más terapéuticos e individuales, avanzando hacia un trabajo colaborativo que permita apoyar más efectivamente, al alumnado con Necesidades Específicas de Apoyo Educativo (de aquí en adelante NEAE) (Acosta et al., 2010). 
Al respecto, el trabajo colaborativo (TC de aquí en adelante) entre diferentes profesionales es un paso fundamental para el desarrollo de la inclusión educativa (Echeita \& Sandoval, 2002; S. Stainback \& W. Stainback, 1999), dado que en la actualidad, ningún profesional cuenta con todos los conocimientos o experiencias para abordar los requerimientos y demandas de los alumnos que participan en aulas abiertas a la diversidad. (ASHA, 2016; Suleman et al., 2014). En este sentido, el TC puede ser una herramienta que permita favorecer la inclusión educativa del alumnado con NEAE en los colegios (Ainscow \& Howes, 2007; Bonilla et al., 2019; Gallagher et al., 2020; Halinen \& Järvinen, 2008). Sin embargo, estos desafíos que representa la inclusión educativa y el TC han problematizado el rol de los profesionales de apoyo como el logopeda (Acosta, 2005; Castejón \& España, 2004).

En la literatura se entiende por TC a las acciones que un grupo de dos o más personas (Friend \& Cook, 1990; Swenson, 2000) realizan en conjunto para favorecer el desarrollo de aprendizajes significativos de todo el alumnado (Nelter, 2014; Swenson, 2000; Welch, 1998). Este tipo de colaboración se ve influenciada por variables culturales (Kochhar-Bryant, 2008; Welch, 1998), personales y organizativas (Hartas, 2004) y puede transitar por niveles de menor a mayor desarrollo (Castejón \& España, 2004; Suleman et al., 2014). Así por ejemplo, Acosta et al. (2010) plantea que dicho TC se condicionaría por distintas variables, relacionadas tanto al tipo de práctica colaborativa, como a los resultados y avances obtenidos por el alumnado.

Desde otra perspectiva, el TC podría considerarse como una herramienta que favorecería el enriquecimiento curricular (Ehren, 2000; Goddard et al., 2007; Swenson, 2000), además de brindar múltiples beneficios, como la adquisición de nuevos conocimientos de parte de los distintos profesionales que se desempeñan en las comunidades educativas. Asimismo, el TC impactaría positivamente en las prácticas pedagógicas (Antúnez, 1999; Neltner, 2014), permitiendo a los profesionales utilizar un lenguaje común (Ehren, 2000) y propiciar ambientes de aprendizaje más auténticos (Neltner, 2014), que beneficiarían a los alumnos que se encuentra dentro en el aula (ASHA, 2016; Glover et al., 2015; Jago \& Radford, 2017; Suleman et al., 2014; Swenson, 2000)

Sin embargo, estas prácticas colaborativas no siempre llegan a desarrollarse o su adscripción puede presentar múltiples desafíos. Al respecto, la literatura ha identificado la existencia de barreras que afectan su desarrollo y que impactarán finalmente, en el tipo de enseñanza recibida por los estudiantes con NEAE, en comparación al resto de sus compañeros (Felón et al., 2010).

Con relación a esto y a pesar de los beneficios que otorga el desarrollo de TC, dichos profesionales no logran desarrollar los niveles esperados en contextos inclusivos (Ehren, 2000; McCartney et al., 2011), dada las distintas barreras a las que se ven enfrentados.

McCartney (1999) plantea que existirían distintas barreras que afectarían el desarrollo de TC entre maestro y logopeda, las cuales podrían agruparse en diferentes niveles. Por ejemplo, Glover et al. (2015) y Hartas (2004) proponen que el TC debe ser mirado desde una perspectiva individual, aludiendo a los conocimientos y habilidades de los profesionales. Desde una perspectiva más institucional, se señala que debe incluirse el lugar o contexto en el que se desarrollan las relaciones de colaboración entre los profesionales, considerando además, a distintos actores educativos como los directivos y el resto de los agentes educativos del colegio (Castejón \& España 2004). Y por 
último, en un nivel más global, deberían ser considerados los distintos lineamientos de los colegios, así como los recursos para el desarrollo de TC (Castejón \& España 2004; Easen et al., 2000; Lindsay \& Dockrell, 2002).

Es necesario destacar que hasta donde sabemos en la literatura disponible no se ha sistematizado la información sobre las barreras que afectan el desarrollo de la colaboración entre maestro y logopeda en escuelas inclusivas. Por lo que resulta un desafío organizar el conocimiento científico en torno a las barreras que perciben los profesionales para la colaboración. Sistematizar las barreras podría facilitar el acceso a la información a quienes toman decisiones políticas para el desarrollo de una educación inclusiva. Por lo tanto, el objetivo de este estudio es realizar una revisión sistemática sobre las barreras que afectan el TC entre maestro y logopeda para posteriormente poder organizarlas en los niveles macro, meso y microsistema desde la perspectiva ecológica, utilizando como marco, el modelo ecológico (Bronfenbrenner \& Morris, 2006). Para esta revisión se plantearon las siguientes preguntas:

1. ¿Qué barreras para el desarrollo de TC han identificado los maestros y logopedas en contextos educativos inclusivos?

2. ¿Cómo se pueden distribuir las barreras declaradas para el TC entre maestros y logopedas, considerando la perspectiva ecológica?

\section{Método}

Para lograr cumplir con el objetivo de la presente revisión, se cumplió con los estándares de revisiones sistemáticas y metaanálisis (PRISMA) (Moher et al., 2010).

$\mathrm{Al}$ respecto, se realizaron búsquedas exhaustivas en las bases de datos electrónicas Web of Science, Scopus, ProQuest, Ebsco, y Scielo durante enero de 2020. La búsqueda se restringió a artículos publicados en inglés y español sin restricciones en el tiempo de publicación. La estrategia de búsqueda incluyó las siguientes palabras claves: Teacher*, collaboration, speech, language, therapists, inclusive education, special education y barriers. Se utilizaron los operadores booleans AND y OR para combinarlas. La técnica de muestreo de "bola de nieve" (snowballing) se utilizó para acceder a estudios relevantes que podrían haber sido publicados en otras bases de datos.

Por otro lado, y considerando que para las revisiones sistemáticas resulta primordial centrar las búsquedas en un grupo de resultados que cumplan con criterios establecidos y claros (ver discusión en COSMIN), los artículos se seleccionaron de acuerdo con los siguientes criterios de inclusión y exclusión: (1) Los estudios debían ser empíricos y se excluyeron, notas editoriales, disertaciones de tesis, los estudios teóricos y documentales. (2) Los estudios debían haberse realizado en el ámbito educativo que, cumpliendo con la perspectiva educacional inclusiva, admiten alumnos con necesidades específicas de apoyo educativo (NEAE). (3) Los estudios debían centrar su atención en el TC entre maestros y logopeda, por lo que excluimos los que abordaron el TC entre otros profesionales donde no estuviera incluida la dupla maestro-logopeda. Mediante las búsquedas electrónicas se identificaron 217 artículos (ProQuest 28\%, Scopus 32\%, Ebsco 18\%, Web of Science 22\%) (Ver Fig. 1). Tras la eliminación manual de duplicados, un evaluador clasificó los títulos y resúmenes de 115 artículos de acuerdo con los criterios de inclusión y exclusión. Posteriormente y como mecanismos de validación, 
un segundo evaluador que no conocía las evaluaciones del primer investigador revisó una muestra aleatoria del $20 \%$ de los artículos. Esta es una práctica recomendable cuando los recursos son limitados y se utilizan muchas bases de datos electrónicas (Pettigrew \& Roberts, 2005).

La doble revisión resultó en un acuerdo de (92.3\%). Los artículos donde las calificaciones difirieron fueron discutidos y se alcanzó un acuerdo de consenso para la mayoría de los documentos. Se consultó a un tercer evaluador para los documentos que quedaron en disputa.

En paralelo, dos evaluadores independientes extrajeron de los artículos seleccionados en la síntesis cuantitativa la siguiente información: autor(es) y año, indexación, nombre de la revista, profesionales considerados, naturaleza, tamaño de muestra y métodos utilizados. Finalmente, y siguiendo el modelo ecológico de Bronfenbrenner, se identificaron las barreras de la colaboración entre maestros y logopedas, las cuales se distribuyeron en los niveles micro-meso-macro que forman parte del modelo ecológico.

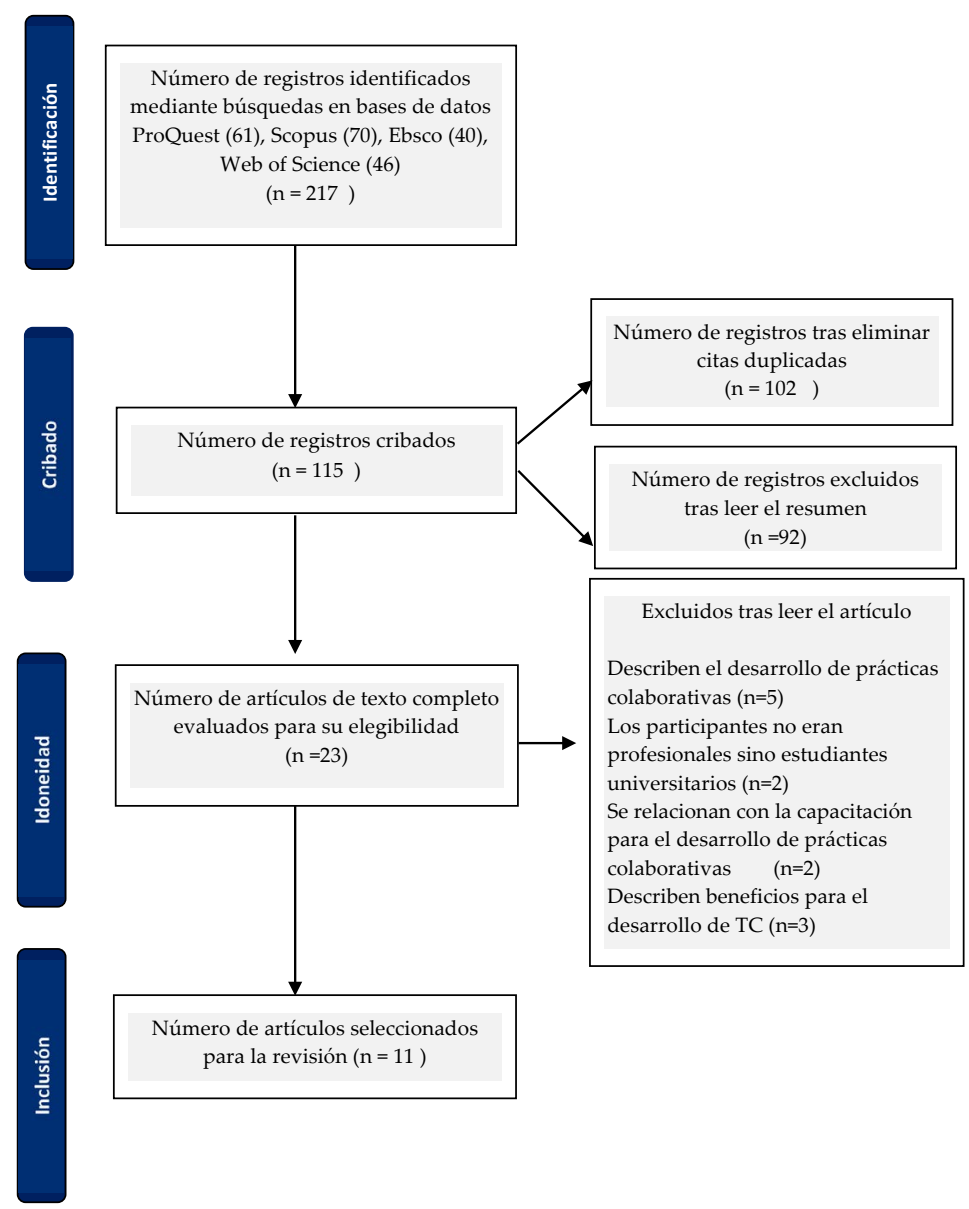

Figura 1. Diagrama de flujo según PRISMA. Fuente: Elaboración propia 


\section{Resultados}

A continuación, se presentan los resultados de la revisión realizada organizadas en función de las dos preguntas planteadas.

En relación con la primera pregunta vinculada al tipo de barreras descritas en el trabajo colaborativo entre logopedas y maestros, la Tabla 1 presenta un resumen de los artículos incluidos en esta revisión. La información se presenta considerando autores, indexación, tipo de estudio, tamaño muestral, características de la muestra e instrumentos de recolección de información.

Tabla 1.

Sintesis de investigaciones revisadas.

\begin{tabular}{llll}
\hline Autor(es) y año Indexación & $\begin{array}{c}\text { Tipo de } \\
\text { estudio/nivel de } \\
\text { profundidad }\end{array}$ & Muestra & Instrumento \\
\hline
\end{tabular}

58 participantes pertenecientes a colegios es-

$\begin{array}{ccc}\text { Kersner y } & \text { Cuantitativo } \\ \text { Wright (1996) } & \text { Scopus } & \text { (Descriptivo) }\end{array}$
peciales de Inglaterra y Wales con usuarios entre 2 y 19 años. Maestros: 26

Logopedas: 32 41 participantes pertenecientes a los distritos oeste de Nueva York.

Nochajski (2002) Scopus $\quad \begin{gathered}\text { Cualitativo } \\ \text { (Descriptivo) }\end{gathered}$
Educadores regulares: 17 Educadores especiales: 12 Terapeutas ocupacionales: 7 Psicólogos:7 Logopeda: 8

Cualitativo

Tollerfield (2003) Proquest (Elaboración de categorías y análisis descriptivos)

Hartas (2004) Scopus $\quad \begin{aligned} & \text { Cuantitativo } \\ & \text { (descriptivo) }\end{aligned}$

\section{3 participantes Logopedas: 2 Maestror:1}

42 participantes pertenecientes a colegios especiales en la ciudad de Midlands Maestros: 25 Logopedas 17
Cuestionario con preguntas cerradas, abiertas y de opciones múltiples

Entrevista semiestructurada:

Compuesta por 5 preguntas orientadoras

Notas de campo Transcripciones de prácticas de audio y video de clases y sesiones de lluvia de ideas

Cuestionario con preguntas cerradas con opciones múltiples, con escala y abiertas 


\begin{tabular}{|c|c|c|c|c|}
\hline Autor(es) y año & Indexación & $\begin{array}{c}\text { Tipo de } \\
\text { estudio/nivel de } \\
\text { profundidad }\end{array}$ & Muestra & Instrumento \\
\hline $\begin{array}{c}\text { Baxter et al. } \\
\text { (2009) }\end{array}$ & $\begin{array}{l}\text { Web of } \\
\text { science }\end{array}$ & $\begin{array}{c}\text { Cuantitativo } \\
\text { (Descriptivo-com- } \\
\text { parativo) }\end{array}$ & $\begin{array}{c}\text { Profesionales de } \\
\text { colegios } \\
\text { Maestros: } 34 \\
\text { Maestros especialista: } \\
30 \\
\text { Coordinador de edu- } \\
\text { cación especial: } 11 \\
\text { Maestros de audición: } \\
10 \\
\text { Maestros practicantes: } \\
10\end{array}$ & $\begin{array}{l}\text { Cuestionario con } \\
\text { preguntas cerradas, } \\
\text { abiertas y escala } \\
\text { Likert }\end{array}$ \\
\hline \multirow[t]{2}{*}{$\begin{array}{l}\text { Kollia y Mulrine } \\
\qquad(2014)\end{array}$} & Proquest & $\begin{array}{l}\text { Cuantitativa (Des- } \\
\text { criptivo) }\end{array}$ & $\begin{array}{l}55 \text { participantes perte- } \\
\text { necientes a } 5 \text { colegios } \\
\text { públicos de la ciudad } \\
\text { de New Jersey } \\
\text { Logopedas: } 17 \\
\text { Maestros generales: } 38\end{array}$ & $\begin{array}{l}\text { Cuestionario con } \\
\text { preguntas de res- } \\
\text { puesta de opción } \\
\text { múltiple y abiertas }\end{array}$ \\
\hline & & & $\begin{array}{l}9 \text { participantes, distri- } \\
\text { buidos en } \\
4 \text { colegios }\end{array}$ & \\
\hline $\begin{array}{l}\text { Pampoulou } \\
\text { (2016) }\end{array}$ & $\begin{array}{l}\text { Web of } \\
\text { science }\end{array}$ & $\begin{array}{c}\text { Cualitativo } \\
\text { (Análisis de conte- } \\
\text { nido) }\end{array}$ & $\begin{array}{c}\text { Maestros especialis- } \\
\text { tas: } 4 \\
\text { Logopedas: } 2 \\
\text { Directivo: } 1 \\
\text { Asistentes de Logope- } \\
\text { das: } 2\end{array}$ & $\begin{array}{l}\text { Entrevista semi- } \\
\text { estructurada }\end{array}$ \\
\hline $\begin{array}{l}\text { Glover et al. } \\
\text { (2015) }\end{array}$ & $\begin{array}{l}\text { Web of } \\
\text { science }\end{array}$ & $\begin{array}{c}\text { Fase 1: } \\
\text { Cuantitativo } \\
\text { Análisis descrip- } \\
\text { tivo } \\
\text { Fase 2: } \\
\text { Cualitativo } \\
\text { Análisis temático }\end{array}$ & $\begin{array}{c}\text { Participantes perte- } \\
\text { necientes a colegio de } \\
\text { primaria. } \\
\text { Fase } 1 \\
\text { Maestros: } 14 \\
\text { Logopedas: } 6 \\
\text { Fase } 2 \\
\text { Maestros: } 1 \\
\text { Logopeda: } 2 \\
\text { Profesional de apoyo: } \\
1\end{array}$ & $\begin{array}{c}\text { Fase 1: } \\
\text { Cuestionario con } \\
\text { preguntas abiertas } \\
\text { y cerradas } \\
\text { Fase 2: } \\
\text { grupo focal }\end{array}$ \\
\hline $\begin{array}{c}\text { Jago y Radford } \\
\text { (2017) }\end{array}$ & $\begin{array}{l}\text { Web of } \\
\text { science }\end{array}$ & $\begin{array}{c}\text { Cualitativo } \\
\text { (Análisis temático) }\end{array}$ & $\begin{array}{l}10 \text { Logopedas } \\
\text { pertenecientes a co- } \\
\text { legios de primaria y } \\
\text { secundaria }\end{array}$ & $\begin{array}{l}\text { Entrevista semi- } \\
\text { estructurada con } 6 \\
\text { preguntas guías }\end{array}$ \\
\hline
\end{tabular}




\begin{tabular}{ccccc}
\hline Autor(es) y año & Indexación & $\begin{array}{c}\text { Tipo de } \\
\text { estudio/nivel de } \\
\text { profundidad }\end{array}$ & Muestra & Instrumento \\
\hline $\begin{array}{c}\text { Tracy-Bronson } \\
\text { et al. (2019) }\end{array}$ & $\begin{array}{c}\text { Web of sci- } \\
\text { ence }\end{array}$ & $\begin{array}{c}\text { Cualitativo } \\
\text { (Análisis de con- } \\
\text { tenido de entre- } \\
\text { vistas) }\end{array}$ & $\begin{array}{c}\text { Colegios pre escolares } \\
\text { Psicólogos: } 4 \\
\text { Terapeutas ocupacio- } \\
\text { nales: } 5 \\
\text { Logopedas: } 6\end{array}$ & $\begin{array}{c}\text { Entrevista semies- } \\
\text { tructurada con 14 } \\
\text { preguntas guías }\end{array}$ \\
$\begin{array}{c}\text { Pfeiffer et al. } \\
\text { (2019) }\end{array}$ & Proquest & $\begin{array}{c}\text { Cuantitativo } \\
\text { (Relacional) }\end{array}$ & $\begin{array}{c}\text { 474 Logopedas que se } \\
\text { desempeñan en edu- } \\
\text { cación }\end{array}$ & Encuesta \\
\hline
\end{tabular}

Fuente: Elaboración propia.

En las investigaciones seleccionadas, se identificaron barreras que afectan el desarrollo de TC. La Tabla 2 ofrece un resumen de las barreras mencionadas por los profesionales.

Tabla 2

Resumen de las barreras que afectan el desarrollo de trabajo colaborativo.

\begin{tabular}{|c|c|c|c|c|c|c|c|}
\hline Niveles & \multirow{3}{*}{ Político } & \multicolumn{5}{|c|}{ Colegio } & \multirow{3}{*}{ Individual } \\
\hline & & \multirow{2}{*}{$\begin{array}{l}\text { Roles y } \\
\text { funciones }\end{array}$} & \multirow{2}{*}{ Comunicación } & \multicolumn{3}{|c|}{ Recursos } & \\
\hline Autores & & & & Tiempo & Humanos & Físicos & \\
\hline $\begin{array}{l}\text { Kersner y } \\
\text { Wright (1996) }\end{array}$ & & & $x$ & & & & $x$ \\
\hline $\begin{array}{l}\text { Nochajski } \\
\text { (2002) }\end{array}$ & & $\mathrm{x}$ & $x$ & $x$ & $x$ & & $\mathrm{x}$ \\
\hline $\begin{array}{c}\text { Tollerfield } \\
\text { (2003) }\end{array}$ & & & & & & & $x$ \\
\hline Hartas (2004) & $x$ & $x$ & $x$ & $x$ & & $x$ & $x$ \\
\hline $\begin{array}{c}\text { Baxter et al. } \\
(2009)\end{array}$ & & $x$ & $x$ & & $x$ & & \\
\hline $\begin{array}{c}\text { Kollia y Mulri- } \\
\text { ne (2014) }\end{array}$ & & $x$ & & $x$ & & & \\
\hline $\begin{array}{c}\text { Pampoulou } \\
(2016)\end{array}$ & & $\mathrm{x}$ & & $\mathrm{x}$ & & & \\
\hline $\begin{array}{c}\text { Glover et al. } \\
(2015)\end{array}$ & $x$ & $x$ & $x$ & $x$ & $x$ & & \\
\hline $\begin{array}{c}\text { Jago y Radford } \\
(2017)\end{array}$ & & $x$ & & $\mathrm{x}$ & & & $x$ \\
\hline $\begin{array}{c}\text { Tracy-Bronson } \\
\text { et al. (2019) }\end{array}$ & & $x$ & & $\mathrm{x}$ & & & $x$ \\
\hline $\begin{array}{c}\text { Pfeiffer et al. } \\
(2019)\end{array}$ & & & & $x$ & & & $\mathrm{x}$ \\
\hline
\end{tabular}

Fuente: Elaboración propia 
Por otra parte, y considerando la segunda pregunta relacionada con la necesidad de organizar la información utilizando el modelo ecológico (Bronfenbrenner \& Morris, 2006), la figura 2 permite observar las distintas barreras ordenadas de acuerdo con los niveles micro, meso y macrosistema respectivamente.

En el Microsistema se agruparon barreras correspondientes a un nivel Individual, relacionadas con características profesionales y personales tanto del maestro como del logopeda. Así, siete de las once investigaciones seleccionadas mencionaron barreras relativas a características de los profesionales que afectarían la colaboración entre ambos. Una de las barreras en este nivel, se relaciona con las diferencias en cómo se entiende el apoyo del alumnado con NEAE, que impactaría directamente en el desarrollo de las practicas colaborativas (Nochajski, 2002; Tollerfield, 2003). Así por ejemplo, en algunas de las investigaciones, los profesionales aludieron a que estas dificultades surgirían cuando no se comparten las mismas ideas o creencias sobre el TC, existiendo por tanto, posiciones contradictorias que afectarían directamente la colaboración (Hartas, 2004; Kersner \& Wright, 1996).

En otras, los participantes de las investigaciones en cuestión, manifestaron la falta de compromiso hacia el TC por parte de distintos actores educativos, tanto a nivel profesional (Kersner \& Wright, 1996), como directivo (Hartas, 2004; Pfeiffer et al., 2019). Esto implicaría una resistencia al cambio de prácticas profesionales colaborativas (Pfeiffer et al., 2019; Tracy-Bronson et al., 2019). Las razones aludidas para esta falta de compromiso fue la percepción que el TC les generaba una pérdida de autonomía e identidad profesional (Hartas, 2004; Kersner \& Wright, 1996; Nochajski, 2002) , al tener que compartir responsabilidades y trabajar en equipo (Hartas, 2004).

Finalmente, desde el plano personal, se identificó que las características personales, actitudes (Jago \& Radford., 2017; Nochajski, 2002) y habilidades sociales, como por ejemplo, la capacidad de comunicar, podían afectar el desarrollo de TC (Hartas, 2004).

En el nivel Mesosistema, se consideraron barreras relacionadas al Colegio. Los once artículos seleccionados en esta revisión mencionaron barreras propias del funcionamiento del colegio que afectan el desarrollo de TC. Por ejemplo, en algunas de ellas, los profesionales entrevistados reconocían que una débil cultura interdisciplinaria (Hartas, 2004), con bajo apoyo administrativo para el desarrollo de prácticas inclusivas (Nochajski, 2002; Tracy-Bronson et al., 2019) y estructuras administrativas rígidas y fijas (Hartas, 2004), impactaban negativamente el desarrollo de TC entre distintos profesionales.

No obstante, y dada la gran variedad de barreras relacionadas al colegio, se organizaron categorías para facilitar su comprensión, estableciéndose como eje central, la Gestión de los Directivos, considerando que la mayoría de la literatura revisada reportó el rol central de esta gestión para promover prácticas colaborativas (Jago \& Radford 2017; Kersner \& Wright, 1996). De esta manera, las categorías finales en este nivel fueron: Roles y Funciones, Comunicación; y Recursos. 
- Roles y Funciones: Ocho de las once investigaciones revisadas reportaron barreras relacionadas con esta categoría. De acuerdo a Glover et al. (2015), Hartas (2004), Jago y Radford (2017) y Tracy-Bronson et al. (2019), maestros y logopedas consideraron que la colaboración se redujo cuando no existía definición de los Roles y Funciones en los miembros del equipo. Asimismo, Baxter et al. (2009), Jago y Radford (2017), Nochajski (2002) y Pampoulou (2016), agregaron que no basta solo con definir los Roles y Funciones, sino que además sería fundamental socializarlos con todos los miembros del equipo. Los profesionales entrevistados reconocían que la poca claridad de sus roles y funciones genera dificultades para la definición de los objetivos (Jago \& Radford, 2017), limitando brindar un adecuado servicio educativo (Baxter et al., 2009), y por tanto, provocado un desajuste entre las metas académicas y terapéuticas para el cumplimiento del currículum escolar (Kollia \& Mulrine, 2014).

- Comunicación: Cinco de los once artículos informaron dificultades en la comunicación (Hartas, 2004; Kersner \& Wright, 1996; Nochajski, 2002), atribuyendo la falta o inexistencia de canales otorgados por el colegio, lo que dificultaba la interacción entre profesionales (Baxter et al., 2009; Glover et al., 2015). .

- Recursos: En esta categoría se mencionaron barreras relacionadas con la disponibilidad de Tiempo, con Recursos Humanos y Físicos. Con respecto a las barreras de Tiempo, ocho de las once investigaciones mencionaron el tiempo como una variable relevante para la colaboración, reconociendo que la práctica cooperativa y de trabajo en equipo es demandante (Nochajski, 2002), y que la escasez del recurso tiempo limitaría el TC (Glover et al., 2015; Hartas, 2004; Jago \& Radford, 2017; Pampoulou, 2016; Pfeiffer et al., 2019). Sin embargo, varias conclusiones respecto a esta variable, plantearon que una mayor disponibilidad de tiempo no garantizaría el TC, dado que esta variable dependería finalmente de la gestión del mismo y de contar con temporalidades comunes entre ambos profesionales, para destinarlo al trabajo conjunto (Kollia \& Mulrine, 2014; Tracy-Bronson et al., 2019). Por otro lado, tres de las investigaciones mencionaron barreras relacionadas a los Recursos Humanos. Glover et al. (2015), Nochajski (2002) y Baxter et al. (2009), reconociendo que la falta de Recursos afectaría el desarrollo de la colaboración, destacándose en la mayoría de los casos, la poca permanencia del logopeda en los colegios, implicaría una inestabilidad en la conformación de los equipos (Jao \& McDougall, 2016). En cuanto a los Recursos Físicos, Hartas (2004) consideró que la falta de espacios de reunión entre maestros y logopedas, así como el material de trabajo para evaluar e intervenir al alumnado, también reducían las posibilidades de desarrollar prácticas colaborativas.

Finalmente, relacionadas al Macrosistema, dos investigaciones de las diez revisadas (Hartas, 2004 y Glover et al., 2015) mencionan barreras relacionadas con este sistema. Particularmente, Hartas (2004) mencionó que la colaboración entre maestros y logopedas se ha visto afectada por la falta de políticas centrales que favorezcan el desarrollo del TC. Glover et al. (2015) por su parte, se refirió a fallas generales en el sistema de financiamiento que generarían dificultades en el desarrollo de la colaboración y listas de espera en la atención del alumnado con NEAE. 


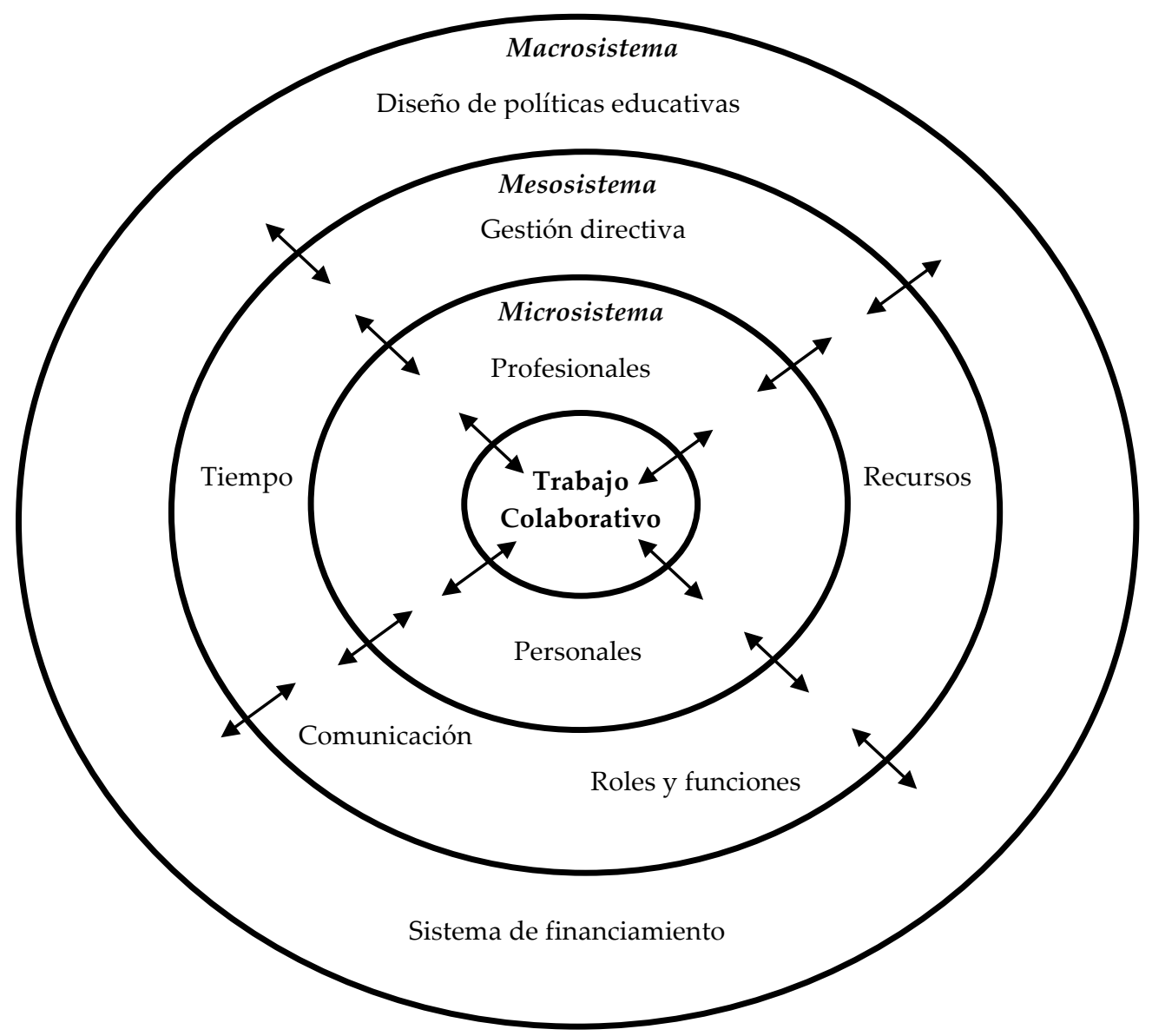

Figura 2. Organización de las barreras que afectan el desarrollo de TC entre maestro y logopeda. Fuente: Elaboración propia

\section{Discusión y Conclusiones}

El objetivo de este estudio fue identificar y organizar las barreras percibidas por maestros y logopedas para el desarrollo de TC en educación, en distintas investigaciones. Para ello, las barreras se organizaron a partir de los niveles micro-meso-macro propuestos en el modelo ecológico (Bronfenbrenner \& Morris, 2006). De esta manera, se logró identificar que la colaboración entre ambos profesionales estaría incidida por variables distribuidas en diferentes niveles del sistema, coincidiendo con los propuesto por McCartney (1999) y destacándose la multiplicidad de factores que impactan en las prácticas de colaboración entre los profesionales .

En primer lugar y referente al nivel Microsistema asociado al nivel Individual, se destaca que la colaboración debe ser experimentada por ambos profesionales como 
una herramienta que les permita resolver los requerimientos, tanto curriculares como terapéuticos dentro del aula. Se reconoce así, que los maestros y logopedas por sí solos no cuentan con el conocimiento y experiencia suficiente para dar respuesta a los diversos requerimientos del alumnado con NEAE (Suleman et al., 2014). Ambos deben beneficiarse de la colaboración y entenderla como una herramienta que les permita compartir conocimientos y experiencias sobre perspectivas y enfoques acerca de cómo dar respuestas educativas a la diversidad de alumnos (Castejón \& España, 2004).

En contextos inclusivos el maestro y logopeda deben involucrarse recíprocamente en el logro de metas escolares del alumnado con y sin NEAE (Nochajski, 2002; Swenson, 2000), ambos deben poder superar las barreras desde el nivel individual (Castejón \& España, 2004; Glover et al., 2015; Lindsay \& Dockrell, 2002). El maestro debe entender el TC como una herramienta que le permitirá trabajar adecuadamente en contextos inclusivos (AEDNEE, 2011b), siendo necesario, que los profesionales puedan compartir y reconocer la importancia, los propósitos y valores sobre la colaboración (Easen et al., 2000).

La investigación de Pfeiffer et al. (2019) señala que un bajo porcentaje de logopedas ha recibido capacitación en colaboración interprofesional durante sus estudios universitarios. Coincidiendo con esto, Wilson et al. (2015) plantean que los estudiantes de logopedia no han desarrollado, en su formación profesional, una apreciación sobre la complejidad de la colaboración con otros profesionales. En su formación profesional presentan pocas instancias de experiencias interprofesionales, por lo que una vez insertos laboralmente, la educación interprofesional (EIP) sobre TC puede ser una oportunidad para superar las barreras al desarrollo de colaboración en el plano individual. En este sentido, Suleman et al. (2014) describen una experiencia de EIP como un buen camino para la formación de profesionales que trabajarán en contextos inclusivos. Los profesionales que durante sus estudios han recibido EIP se muestran más dispuestos a aplicar diferentes modelos colaborativos. Esta investigación coincide con los resultados planteados por Vega et al. (2020), donde maestros que vivieron procesos de asesoramiento colaborativo, mejoraron sus concepciones sobre la importancia del desarrollo de TC en el proceso de enseñanza.

La EIP para los diferentes actores del sistema educativo, es una herramienta que permitirá a los maestros y logopedas a mejorar la colaboración, fomentando gradualmente una cultura colaborativa dentro sus respectivos colegios (Pfeiffer et al., 2019). La EIP también debe considerarse en los procesos formativos de ambos profesionales, en el cual se deberían abordar temáticas en común como el desarrollo de prácticas colaborativas con otros profesionales (Sinai-Gavrilov et al., 2019; Wilson et al., 2016).

En segundo lugar y relacionado con el Mesosistema a nivel de Colegio, las barreras que afectan el TC dependen en gran medida de la gestión pro colaboración que realizan los directivos (Dockrell et al., 2014). De ellos depende que en el colegio se desarrolle un compromiso colectivo a favor de la colaboración, construyendo una trama de relaciones humanas positivas que favorezcan la inclusión escolar (Castejón \& España, 2004). Según Pfeiffer et al. (2019) la falta de apoyo por parte de los directivos o empleadores, generaría dificultades para alcanzar mayores niveles de colaboración. Al respecto, el liderazgo de los directivos a nivel escolar juega un papel importante, ya que cuando comprenden y promueven el TC, la comunidad educativa en general alcanza mayores 
niveles de colaboración (Pampoulou, 2016). Los directivos deben tener una clara visión de cómo promover una cultura colaborativa en sus colegios que permitan superar las barreras y mejorar las experiencias escolares de todo el alumnado (Unión Europea, 2017). Un paso importante para lograr mayor desarrollo de TC, es definir y socializar los Roles y Funciones, tanto de los maestros, logopedas y del equipo de trabajo en general. Cuando los directivos logran la asignación de responsabilidades de colaboración, generan instancias para compartir experiencias y visiones profesionales, conciliando perspectivas académicas y terapéuticas para apoyar al alumnado con NEAE (Glover et al., 2015). Cuando ambos profesionales comprenden su rol dentro del equipo, los directivos pueden propiciar el desarrollo de una buena comunicación, permitiendo el tránsito hacia una cultura más colaborativa, privilegiando el diálogo y la reflexión (ASHA, 2001; Castejón \& España, 2004)

Otra barrera mencionada en el Mesosistema corresponde a los Recursos, particularmente la disponibilidad de tiempo para colaborar. En general, los profesionales consultados en las investigaciones, relevaron que la falta de horas de contrato para el TC, así como la poca permanencia en el colegio, serían las causas para impedir prácticas de colaboración (Castejón \& España, 2004; McCartney, 1999). En este sentido, profesionales educativos como los logopedas, que por lo general y dependiendo de la normativa de cada país, cuentan con condiciones laborales que no necesariamente les permitirían contar con horas dedicadas por contrato. Por último y continuando con el grupo de barreras del Mesosistema, la gestión de los directivos sería clave para la promoción y gestión adecuada del tiempo, garantizando los espacios de reuniones entre los profesionales, permitiendo instancias de planificación de las actividades académicas (Sinai-Gavrilov et al., 2019)..

En el Macrosistema, las barreras agrupadas en el nivel político sugieren que el TC debe ser guiado a través de políticas educativas que puedan responder a las necesidades de la comunidad escolar (Castejón \& España, 2004; Easen et al., 2000). Tanto el diseño de políticas educativas (Cartwright y Howick, 2010) como los programas de intervención aplicados al aula para el TC entre maestro y logopeda (Archibald, 2017), deben sustentarse en evidencia científica. Por lo tanto, las políticas educativas deben transitar hacia modelos inclusivos que impliquen TC entre el maestro y otros profesionales como el logopeda. Diversos esfuerzos a nivel políticos reconocen la importancia de la inclusión y del TC, en el caso de España la promulgación de la LOMCE (2013).

En síntesis, esta revisión tiene alcances teóricos que permitirían contribuir a la comprensión de las barreras al TC entre maestros y logopedas en contextos inclusivos. Desde un punto de vista estructural, ofrece un marco conceptual para los profesionales que se desempeñan en educación, proponiendo que el desarrollo del TC requiere de múltiples variables que se pueden organizar en diferentes niveles, que se encuentran en permanente interacción. Pero además, esta revisión podría contribuir a la toma de decisiones en políticas públicas, directivos escolares y profesionales, para comprender mejor el TC y por ende, promover estrategias de intervención pro colaboración, orientadas a prácticas más inclusivas en las aulas (UNESCO, 2015).

Por otro lado y considerando los alcances prácticos de este trabajo, se podrían sugerir que los distintos niveles propuestos implican diferentes niveles de responsabilidad en la toma de decisiones y ejecución del TC. Así, ciertas barreras dependerían de caracterís- 
ticas propias de los profesionales, mientras otras de la gestión que realizan los equipos directivos en los colegios, en tanto, e niveles más macro o globales, serían las políticas públicas las encargadas de orientar, regular y promover el TC en las formaciones de los profesionales y en la puesta en marcha en los colegios.

A pesar de la contribución de este estudio, las investigaciones revisadas presentan algunas limitaciones. La primera es que aun cuando se identificaron barreras para el desarrollo del TC, no se ha determinado aun cómo cada una de estas impactan en su desarrollo, lo cual podría facilitar la canalización de los recursos para su revisión y asesoramiento tanto a nivel político, escolar e individual. En segundo lugar, esta revisión podría incorporar otros profesionales, como en el caso de España, el maestro de audición y lenguaje, debido a que junto con el maestro de aula ordinaria y el logopeda deben trabajar colaborativamente para apoyar a alumnado con NEAE. Al respecto, algunas investigaciones sugieren que la percepción de estos profesionales ha establecido que la baja colaboración con el maestro de aula impide lograr una adecuada labor profesional e inclusiva (de la Rosa \& Carrión, 2013), situando más bien su rol, a un modelo clínico que educativo o curricular (Carrión et al., 2013).

Finalmente, para futuras investigaciones sería interesante poder acotar el estudio de las barreras que afectan el desarrollo de TC a una tarea específica desarrollada entre ambos profesionales dentro del aula. Esto permitiría entender y diseñar estrategias de intervención específicas, considerando posibles soluciones para fomentar el logro de mayores niveles de colaboración.

\section{Referencias}

Acosta, V. (2005). Evaluación, intervención e investigación en las dificultades del lenguaje en contextos inclusivos. Revisión, resultados y propuestas. Revista de Logopedia, Foniatría y Audiología, 25(4), 148-161. doi:10.1016/S0214-4603(05)75833-2

Acosta, V. Moreno, A., Axpe, Á., \& Lorenzo, M. (2010). Apoyo al desarrollo de habilidades narrativas en niños con trastorno específico del lenguaje dentro de contextos inclusivos. Revista de Logopedia, Foniatría y Audiología, 30(4), 196-205. doi:10.1016/ S0214-4603(10)70156-X

Agencia Europea para el Desarrollo de la Educación del Alumnado con Necesidades Educativas Especiales (2011a). Principios fundamentales para la promoción de la calidad de la educación inclusiva - Recomendaciones para la puesta en práctica, Odense, Dinamarca.

Agencia Europea para el Desarrollo de la Educación del Alumnado con Necesidades Educativas Especiales (2011b). Formación del profesorado para la educación inclusiva Recomendaciones claves. https://www.european-agency.org/sites/default/files/ te4i-key-policy-messages_es.pdf.

Ainscow, M. (2017). Haciendo que las escuelas sean más inclusivas: lecciones a partir del análisis de la investigación internacional. Revista de Inclusión Educativa, 5(1), 39-49.

Ainscow, M., \& Howes, A. (2007). Working together to improve urban secondary schools: a study of practice in one city. School leadership and management, 27, (3), 285-300.

Antúnez, S. (1999). El trabajo en equipo de los profesores y profesoras: factor de calidad, necesidad y problema. El papel de los directivos escolares. Educar, 24 (1), 89-110. 
American Speech-Language-Hearing Association. (2001). Roles and Responsibilities of Speech-Language Pathologists With Respect to Reading and Writing in Children and Adolescents [Technical Report]. https://www.asha.org/policy.

American Speech-Language Hearing Association. (2016). Interprofessional Education and Interprofessional Practice in Communication Sciences and Disorders. En A. Johnson, P. Prelock \& K Apel, IPE 101: Introduction to Interprofessional Education and Practice for Speech-Language Pathology (pp 1-25)

Archibald, L. M. (2017). SLP-educator classroom collaboration: A review to inform reason-based practice. Autism \& Developmental Language Impairments, 2(1), 1-17. doi.org/10.1177/239694151668036.

Axpe, A., Acosta, V., Moreno, A., \& Ramírez, G. (2017). Application of a lexicalsemantic intervention programme for students with Specific Language Impairment / Aplicación de un programa de intervención léxico-semántica en alumnado con Trastorno Específico del Lenguaje. Cultura y Educación, 29(2), 324-349. doi: 10.1080/11356405.2017.1305073

Baxter, S., Brookes, C., Bianchi, K., Rashid, K., \& Hay, F. (2009). Speech and language therapists and teachers working together: Exploring the issues. Child Language Teaching and Therapy, 25(2), 215-234. doi:10.1177/0265659009102984

Bonilla, M., Fernández, J., \& Vázquez, M. (2019). Diagnóstico del trabajo colaborativo en un centro escolar como indicador de inclusión educativa. RIDE Revista Iberoamericana para la Investigación y el Desarrollo Educativo, 10(19). doi:10.23913/ride.v10i19.518

Bronfenbrenner, U., \& Morris, P. (2006). The Bioecological Model of Human Development. In R. Lerner (eds), Handbook of child psychology sixth edition volume one: theoretical models of human development. (6 ed., pp. 793-827). New Jersey: John Wiley \& Sons, Inc.

Cartwright, N., \& Howick, J. (2010). Evidence-based policy: Where is our theory of evidence?. Journal of Children's Services, 4(4), 6-14. doi:10.5042/jcs.2010.0017

Carrión M, J., Luque de la Rosa, A. y Fernández S. J. (2013) La intervención en audición y lenguaje en los equipos de orientación educativa de Almería (España). Revista Española de Orientación y Psicopedagogía 24(1), 42-61.

Castejón, L., \& España, G. (2004). La colaboración logopeda-maestro: hacia un modelo inclusivo de intervención en las dificultades del lenguaje. Revista de Logopedia, Foniatría y Audiología, 24(2), 55-66. doi:10.1016/s0214-4603(04)75781-2

de la Rosa, A. L. \& Carrión, J. J. (2013). Análisis del grado de satisfacción profesional del maestro de audición y lenguaje itinerante. European Journal of Education $\mathcal{E}$ Psychology. 6(1), 55-68.

Dockrell, J., Lindsay, G., Roulstone, S., \& Law, J. (2014). Supporting children with speech, language and communication needs: An overview of the results of the Better Communication Research Programme. International Journal of Language and Communication Disorders, 49(5), 543-557. doi:10.1111/1460-6984.12089

Easen, P., Atkins, M., \& Dyson, A. (2000). Inter-professional collaboration and conceptualisations of practice. Children and Society, 14(5), 355-367. doi: 10.1111/j.10990860.2000.tb00190.x

Echeita S., \& Sandoval, M. (2002). Educación inclusiva o educación sin exclusiones. Revista de educación, 327(1), 31-48 
Ehren, B. (2000). Maintaining a therapeutic focus and sharing responsibility for student success: Keys to in-classroom speech-language services. Language, Speech, and Hearing Services in Schools, 31(3), 219-229. doi:10.1044/0161-1461.3103.219

Fenlon, A., McNabb, J., \& Pidlypchak, H. (2010). So much potential in reading! Developing meaningful literacy routines for students with multiple disabilities. Teaching Exceptional Children, 43(1), 42-48.

Friend, M., \& Cook, L. (1990). Collaboration as a Predictor for Success in School Reform. Journal of Educational and Psychological Consultation, 1(1), 69-86. doi: 10.1207/ s1532768xjepc0101_4

Gallagher, A., Murphy, C., Conway, F., \& Perry, A. (2020). Establishing premises for inter-professional collaborative practice in school: inclusion, difference and influence. Disability and Rehabilitation, 17(1), 1-10. doi.org/10.1080/09638288.2020.1725154

Glover, A., McCormack, J., \& Smith-Tamaray, M. (2015). Collaboration between teachers and speech and language therapists: Services for primary school children with speech, language and communication needs. Child Language Teaching and Therapy, 31(3), 363-382. doi: 10.1177/0265659015603779

Goddard, Y., Goddard, R., \& Tschannen-Moran, M. (2007). A theoretical and empirical investigation of teacher collaboration for school improvement and student achievement in public elementary schools. Teachers College Record, 109(4), 877-896.

Halinen, I., \& Järvinen, R. (2008). En pos de la educación inclusiva: el caso de Finlandia. Perspectivas, 38(1), 97-127.

Hartas, D. (2004). Teacher and speech-language therapist collaboration: being equal and achieving a common goal?. Child Language Teaching and Therapy Collaboration, 20(1), 33-54. doi: 10.1191/0265659004ct262oa.

Jago, S., \& Radford, J. (2017). SLT beliefs about collaborative practice: Implications for education and learning. Child Language Teaching and Therapy, 33(2), 199-213. doi: $10.1177 / 0265659016679867$

Jao, L., \& McDougall, D. (2016). Moving beyond the barriers: supporting meaningful teacher collaboration to improve secondary school mathematics. Teacher Development, 20(4), 557-573. doi: 10.1080/13664530.2016.1164747

Kersner, M., \& Wright, J. A. (1996). Collaboration between teachers and speech and language therapists working with children with severe learning disabilities (SLD): Implications for professional development. British Journal of Learning Disabilities, 24(1), 33-37. doi: 10.1111/j.1468-3156.1996.tb00196.x

Kollia, B., \& Mulrine, C. (2014). Collaborative Practice Patterns for Included Students among Elementary Educators and Speech and Language Pathologists. Journal of Education and Human Development, 3(4), 33-44. doi:10.15640/jehd.v3n4a3

Koutsoftas, D., Harmon, T., \& Gray, S. (2009). The effect of Tier 2 intervention for phonemic awareness in a Response-to-Intervention model in low-income preschool classrooms. Language, Speech, and Hearing Services in Schools, 40(2), 116-130.

Kochhar-Bryant, C. (2008). Collaboration and system coordination for students with special needs: From early childhood to the postsecondary years. Columbus, Ohio: Pearson, Merrill Prentice Hall.

Ley Orgánica 8/2013, de 9 de diciembre, para la mejora de la calidad educativa. Boletín Oficial del Estado. http://www.stecyl.es/files/LOMCE/LOE-LOMCE.pdf 
Lindsay, G., \& Dockrell, J. (2002). Editorial Meeting the needs of children with speech language and communication needs: a critical perspective on inclusion and collaboration. Child Language Teaching and Therapy, 18(2), 91-101. doi: 10.1191/0265659002ct229ed

McCartney, E. (1999). Barriers to collaboration: An analysis of systemic barriers to collaboration between teachers and speech and language therapists. International Journal of Language and Communication Disorders, 34(4), 431-440. doi: 10.1080/136828299247379

McCartney, E., Boyle, J., Ellis, S., Bannatyne, S., \& Turnbull, M. (2011). Indirect language therapy for children with persistent language impairment in mainstream primary schools: Outcomes from a cohort intervention. International Journal of Language and Communication Disorders, 46(1), 74-82.

Moher, D., Liberati, A., Tetzlaff, J. y Altman, G. (2010). Elementos de informes preferidos para revisiones sistemáticas y metanálisis: la declaración PRISMA. Annals of Internal Medicine, 8 (5), 336-341.

Moreno, A., Axpe, Á., Acosta, V. (2012). Efectos de un programa de intervención en el lenguaje sobre el desarrollo del léxico y del procesamiento fonológico en escolares de Educación Infantil con Trastorno Específico del Lenguaje. Revista de Investigación Educativa, 30 (1), 71-86.

Murphy, A., Franklin, S., Breen, A., Hanlon, M., McNamara, A., Bogue, A., \& James, E. (2016). A whole class teaching approach to improve the vocabulary skills of adolescents attending mainstream secondary school, in areas of socioeconomic disadvantage. Child Language Teaching and Therapy, 33(2), 129-144

Neltner, R. (2014). Exploring Collaboration between Speech- Language Pathologists and Special Educators in Secondary Education Settings. Recuperado de: https:// scholarworks.bgsu.edu/honorsprojects/125

Nochajski, S. M. (2002). Collaboration Between Team Members in Inclusive Educational Settings. Occupational Therapy In Health Care, 15(3-4), 101-112. doi: 10.1080/J003v15n03

Pampoulou, E. (2016). Collaboration between speech and language therapists and school staff when working with graphic symbols. Child Language Teaching and Therapy, 32(3), 361-376. doi: 10.1177/0265659016647996

Pettigrew, M., \& Roberts, H. (2005). Systematic reviews in the social sciences: a practical guide. Victoria: Blackwell.

Pfeiffer, D., Pavelko., Hahs-Vaughn, D., \& Dudding, C. . (2019). A National Survey of Speech-Language Pathologists' Engagement in Interprofessional Collaborative Practice in Schools: Identifying Predictive Factors and Barriers to Implementation. Language Speech and Hearing Services in Schools, 50(4), 639-655. doi: 10.1044/2019_ LSHSS-18-0100

Sinai-Gavrilov, Y., Gev, T., Mor-Snir, I., \& Golan, O. (2019). Seeking Team Collaboration, Dialogue and Support: The Perceptions of Multidisciplinary Staff-Members Working in ASD Preschools. Journal of Autism and Developmental Disorders, 49(11), 4634-4645. doi: 10.1007/s10803-019-04175-x

Stainback, Susan \& Stainback, William. (1999). Aulas Inclusivas. Madrid: NARCEA.

Steele, S., \& Mills, M. (2011). Vocabulary intervention for school-agechildren with language impairment: A review of evidence andgood practice. Child Language Teaching and Therapy, 27(3), 354-370. doi: 10.1177/0265659011412247 
Suleman, S, McFarlane, L, Pollock, K, Schneider, P, Leroy, C, Skoczylas, M. (2014). Collaboration : More than "Working Together" An exploratory study to determine effect of interprofessional education on awareness and application of models of specialized service delivery by student speech-language pathologists and teachers. Canadian Journal of Speech-Language Pathology and Audiology, 37(4), 298-307.

Swenson, N. (2000). Comparing Traditional and Collaborative Settings for Language Intervention. Communication Disorders Quarterly, 22(1), 12-18. doi: $10.1177 / 152574010002200103$

Tollerfield, I. (2003). The process of collaboration within a special school setting: An exploration of the ways in which skills and knowledge are shared and barriers are overcome when a teacher and speech and language therapist collaborate. Child Language Teaching and Therapy, 19(1), 67-84. doi: 10.1191/0265659003ct244oa

Tracy-Bronson, C., Causton, J., \& MacLeod, K. (2019). Everybody Has the Right to Be Here: Perspectives of Related Service Therapists. International Journal of Whole Schooling, 15(1), 132-174.

Unión Europea. (2017). Ec.europa.eu: Pilar Europeo de los derechos sociales. Recuperado de: https://ec.europa.eu/education/policies/european-policy-cooperation/ inclusive-education_es

United Nations Educational, Scientific and Cultural Organization (2015). Educación 2030. Declaración de Incheon. Hacia una educación inclusiva, equitativa y de calidad y un aprendizaje a lo largo de la vida para todos. Declaración de Incheon, 18-36.

Vega, F., Gràcia, M., \& Riba, C. (2020). Collaborative counselling: Influence on the teaching professionals' conceptions as promoters of children's communication and language. Journal of Psychologists and Counsellors in Schools, 30(2), 227-245. doi: 10.1017/jgc.2019.25 .

Welch, M. (1998). The IDEA of collaboration in special education: An introspective examination of paradigms and promise. Journal of Educational and Psychological Consultation, 9(2), 119-142. doi: 10.1207/s1532768xjepc0902_2

Wilson, L., McNeill, B., \& Gillon, G. (2015). The knowledge and perceptions of prospective teachers and speech language therapists in collaborative language and literacy instruction. Child Language Teaching and Therapy, 31(3), 347-362. doi: $10.1177 / 0265659015585374$

Wright, L., Pring, T., \& Ebbels, S. (2018). Effectiveness of vocabulary inter-vention for older children with (developmental) language disorder. International Journal of Language y Communication Disorders, 33(3), 480-494. doi: 10.1111/1460-6984.12361

Fecha de recepción: 21 de septiembre de 2020.

Fecha de revisión: 3 de octubre de 2020.

Fecha de aceptación: 17 de marzo de 2021. 